This document is published in:

Automation in Construction (2011), 20(5), 629-636.

DOI: $10.1016 /$ j.autcon.2010.12.005

(C) 2010 Elsevier B.V.

This work has been supported by the CAM Project S2009/DPI-1559/ROBOCITY2030 II, developed by the research team RoboticsLab at the University Carlos III of Madrid. 


\title{
Robot-aided tunnel inspection and maintenance system by vision and proximity sensor integration
}

\author{
J.G. Victores, S. Martínez, A. Jardón, C. Balaguer \\ Robotics Lab, Engineering Systems and Automation Department, Universidad Carlos III de Madrid, 28911 Leganés (Madrid), Spain \\ E-mail: \{jcgvicto, scasa, ajardon,balaguer\}@ing.uc3m.es
}

\begin{abstract}
This article describes an unprecedented alternative to manual procedures for the application of advanced composite materials, such as Fiber Reinforced Polymer (FRP) and epoxy resins. A complete mobile integrated system is presented for the inspection and maintenance of concrete surfaces in tunnels. It allows performance of operations with minimum interference on passing traffic. The core of this system resides in a specially designed light-weight robotic tool, which is sensed and automated for processes. Sensing includes vision and a laser telemeter to assure precise inspection, superficial preparation, and composite application. The designed interconnection flange allows simple and robust attachment of the tool to a robotic arm's tip. The robot-tool set is to be mounted on a standard articulated lift platform. Therefore, an operator can direct the platform and the robot-tool set's operations from a control station placed at ground-level, in a wheeled vehicle on which the articulated lift platform is mounted. A graphical Human-Machine Interface (HMI) has been developed for the system. It allows the operator to identify fissures for the injection of epoxy resin, and weakened surfaces for FRP adhesion. Actual procedures are planned and performed by the system's automatic components.
\end{abstract}

Keywords: Robotic tool design, Robotic automation, Tunnel maintenance, Concrete inspection, HMI

\section{Introduction}

Tunnels nowadays are designed and built to last hundreds of years. However, change in use, new load criteria, and impact and damage caused by natural and human factors can drastically reduce a tunnel's service life. Moreover, as tunnels throughout the world and in Europe in particular become older, matters of inspection and maintenance adopt an ever increasing degree of importance [1].

Inspection and maintenance operations in tunnels depend heavily upon time and space constraints: the tunnel's intrinsic conditions (reduced space, possible existence of service pipes), traffic flow, the presence of aerial electric cable in railway tunnels. Working conditions in these subterranean infrastructures can be slow and tedious. Dust, humidity, and complete absence of natural light create uncomfortable and at times unhealthy working conditions. Efficiency and stigma of operators gradually reduce throughout the day in these conditions, incrementing the risk of damage, hazard, or incorrectness of procedures. Additionally, in many cases traffic flow must be cut for operations to be performed, as scaffolds must be mounted and security assured. Any attempt to automate operations performed in these subterranean infrastructures will drastically improve short and long-term productivity, as well as supporting operators in their task [2][3].

A great range of factors can cause need for maintenance or reparation operations. Two of the most important of these factors will be treated:
- Fissure formation due to deformation caused by excessive load or caused by bending moments induced by heterogeneous soil and rock conditions [4]. Although current legislations tolerate the existence of small fissures, their real dimensions should never exceed a small, reduced range. Reinforced concrete's interior metal infrastructure should never be exposed to ambient atmosphere.

- Loss of quality of the infrastructure's surface due to lack of correct metal-conglomerate adhesion. This may be caused by external forces that erode the external surface, or by general or local corrosion [5].

\section{Maintenance operations}

Presently, practically all inspection and maintenance operations in tunnels are performed manually. Frequently, traffic flow must be cut, and scaffolds mounted, implying the subsequent loss of global productivity. The maintenance operations studied for automation call for the following set of tasks: superficial preparation, fissure injection, and FRP composite adhesion.

\subsection{Superficial preparation}

This includes all of the processes needed to eliminate concrete in bad state. Loss of mechanical capacity or of stability within the rest of 


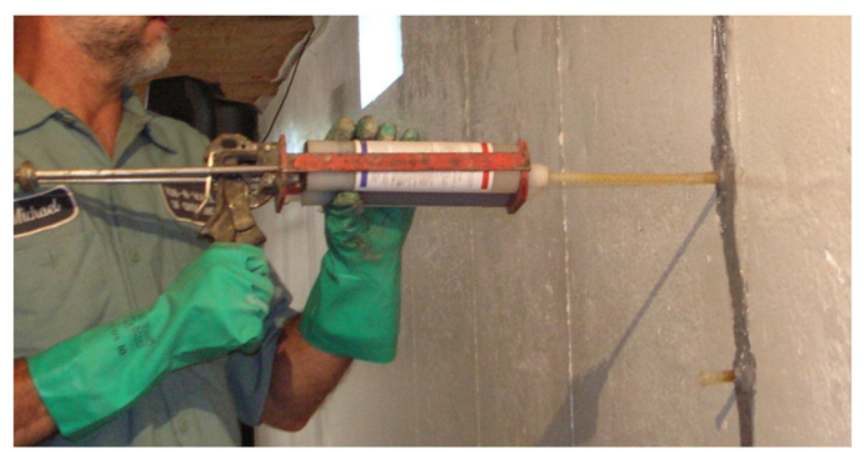

Fig. 1. Conventional manual procedure of fissure injection.

the structure can be a possible reason for this need. The surface must be prepared before concrete reparation and/or restoration. Common methods include compressed air blowing, sand abrasion, and hydrodemolition (preparation with high-pressured water). The final prepared surface must be dry and free from sand and dust.

\subsection{Fissure injection}

Current developments of advanced materials such as low-viscosity epoxy resins allow pressure injection of these materials through fissures as small as $0.2 \mathrm{~mm}$ thick. They are usually thixotropic, solvent-free, and bi-component. Components are included indifferent compartments of a same cartridge and combined through a static mixer at application time (Fig. 1). The main objective of these injections is to re-establish continuity in concrete sections. Injections can be used to fill in internal or hard-to-access zones (such as internal nests). These techniques have been used and tested on tunnels, bridges, and several nuclear centrals over the past few years.

\subsection{FRP composite adhesion}

Since its first applications in Europe and Japan in the 1980s, superficial reparation and restoration with Fiber Reinforced Polymer

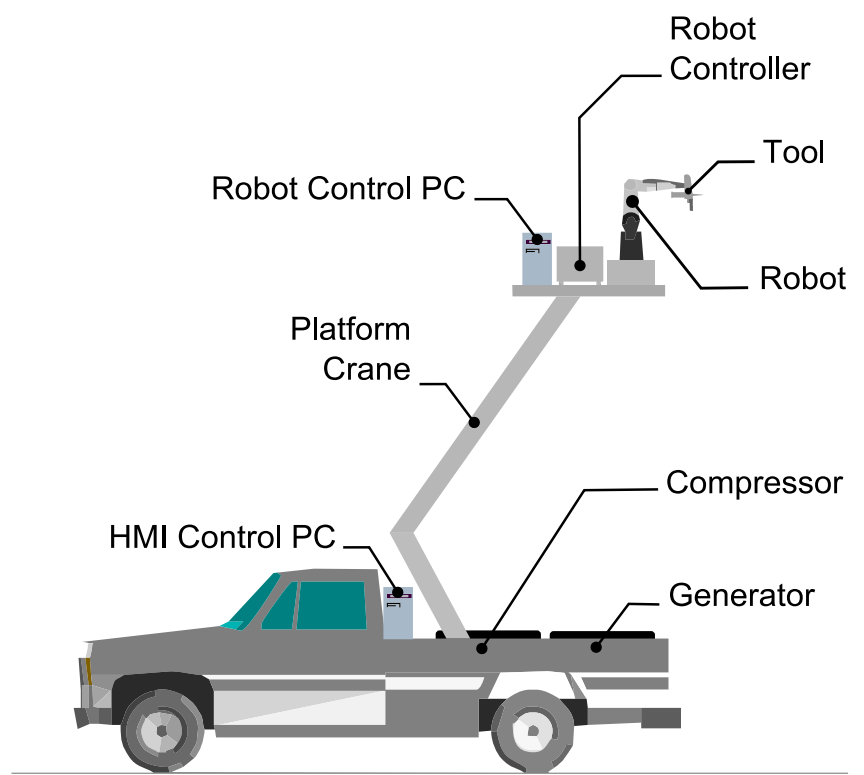

Fig. 3. Basic hardware architecture.

(FRP) has progressively increased. Quick and easy installation and high versatility make FRP reparations attractive to owners, engineers and promoters. FRP adhesion now is also becoming a common practice among automotive, marine, and aerospace industries. Carbon or aramid fibers allow high mechanic, thermal, electric and chemical resistance, high modulus of elasticity, and low density [6].

Technical details and formulae for calculating tensile strength gain by FRP adhesion can be found throughout literature [7]. Commercially available FRP includes rolls that have been pre-impregnated with epoxy resin, and dry rolls.

Basic manual procedures for adhesion of dry-roll FRP can be decomposed into a sequence of three steps, seen in Fig. 2.

In the first step of the sequence, the operator spreads epoxy resin homogeneously on the surface to be treated. This operation is

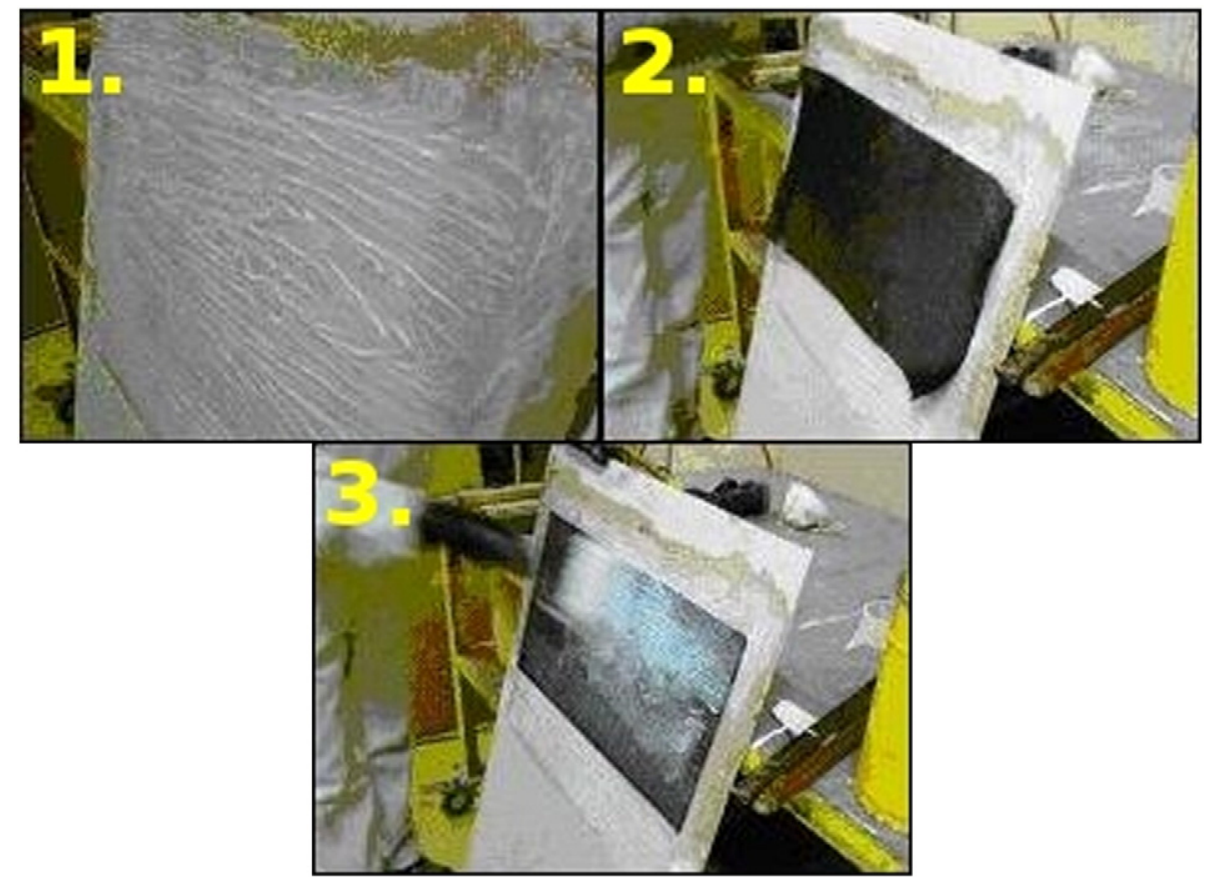

Fig. 2. Conventional manual procedure of FRP composite adhesion. 


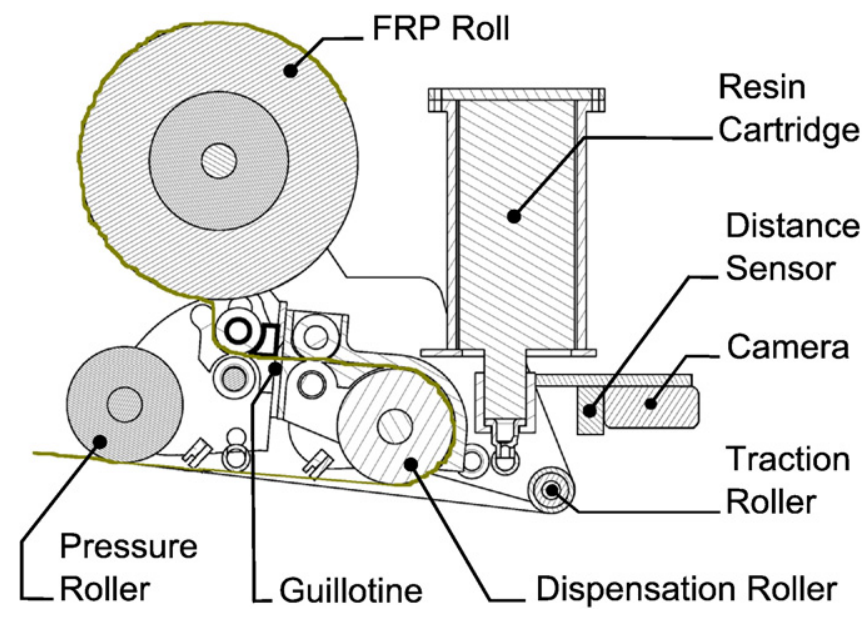

Fig. 4. Tool's basic conceptual design.

performed with a common paintbrush. The applied resin is specific to the FRP and, as bi-component mixture, has been mixed by the operator in a separate pail and must be applied before its curing time.

In the next step, the FRP is laid over the resin-impregnated surface, and is pressed against it with a metallic roller. This roller is hard enough to press the FRP until it gets moist with resin, and its diameter is small enough to adapt to a tunnel's curved surfaces.

After this, a finishing layer of resin is applied. The resin applied in this last layer is of the same type of that of the first one.

\section{Integrated process automation}

The Robot-Aided Tunnel Inspection and Maintenance (RATIM) system is the result of one of the first attempts ever to automate superficial preparation, fissure injection, and FRP composite adhesion procedures, all in one. For this purpose, an integrated system was mounted with a specially designed tool, a robot arm, and a wheeled vehicle with a platform lift (see Fig. 3). Similar configurations have been used recently with Kuka robots in the Hanyang University, South Korea, for different kinds of applications also related with the construction industry [8].

Inspection in the RATIM system is performed through a simple and very intuitive HMI, where the camera image stream is displayed at all times and operation procedures are solicited. Visual servoing is based on images and the depth measure captured by the tool's laser telemeter. Operation-oriented actuators are coordinated through task-specific control software [9].

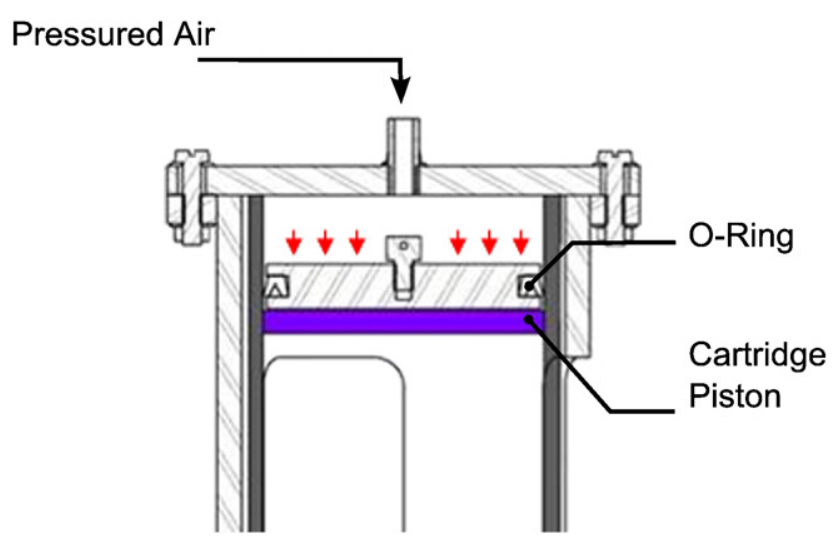

Fig. 5. Resin activation mechanism.

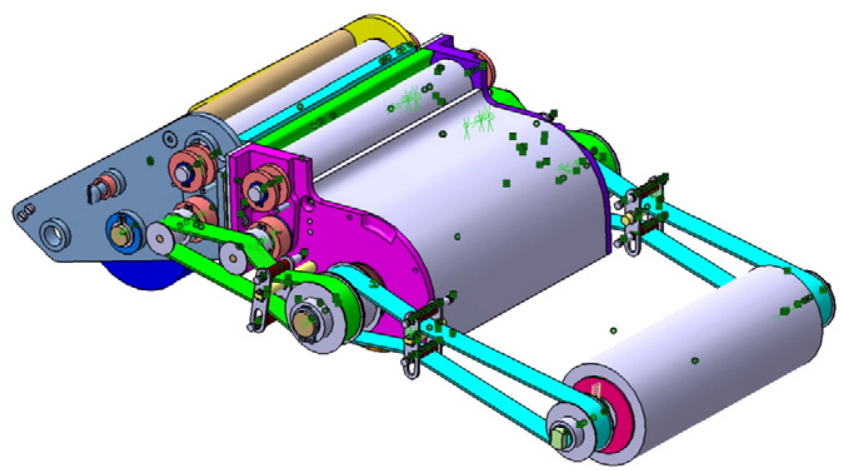

Fig. 6. FRP dispensing mechanism.

For dexterous and light-weight operation, the robot arm chosen for the application was the Mitsubishi PA-10, a 7 DOF manipulator with a $10 \mathrm{~kg}$ load capacity and a 1 meter maximum extension range, weighting only $35 \mathrm{~kg}$ itself [10]. The global increment of this range is achieved by mounting the Robot-Tool set on a 5 meter extensible articulated lift platform. The HMI is installed in the wheeled vehicle's cabinet to which the articulated lift platform is attached. Power for the system can be supplied from an on-board generator, the wheeled vehicle's motor, or the tunnel's basic provided services. When the surface to treat is inside the reach volume, user extends the vehicle's stabilizers, directs the platform towards the concrete surface, and then follows the HMI's on-screen instructions.

\subsection{Specially designed tool}

A light-weight sensed integrated tool was designed and manufactured for the automation of the described processes. Material

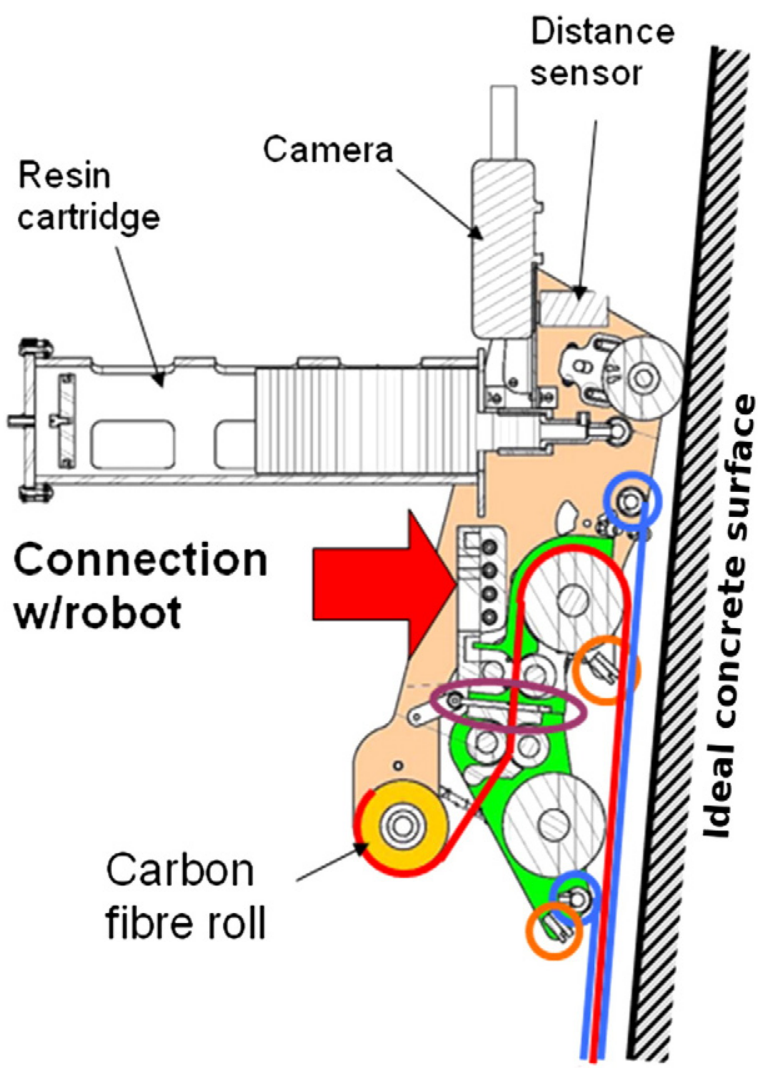

Fig. 7. FRP application scheme. 


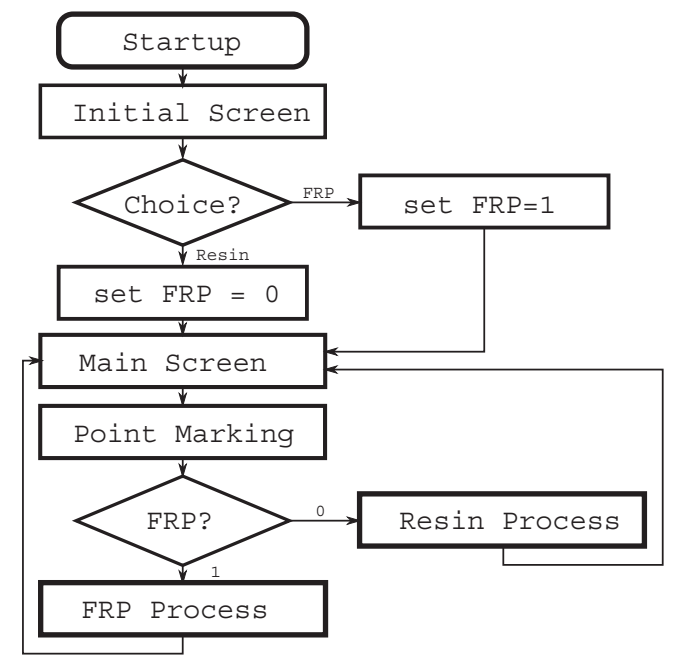

Fig. 8. HMI main program flow diagram.

provider's workshops and real construction sites were visited to analyze manual procedures 'in situ'. These procedures have been described in Section 2 of this article. Conclusions were that radical and innovative approaches would have to be taken to lead this system to complete automation. Main objectives were set as the following:

- Minimum changes should be needed to be effectuated on the tool to change between processes of superficial preparation and resin injection, and processes of superficial preparation and FRP adhesion.

- A single-pass FRP adhesion process is desirable. This implies FRP must be dispensed and pressed against the surface simultaneous to the advance of the tool and release of sufficient resin.

- Maintaining the tool light-weight and compact throughout the design process would allow it to be attached and used within a wide range of commercial and non-commercial robots.

- The robot-tool interconnection flange must allow simple mounting and demounting. Vision, laser distance, and security systems should be intrinsic to the tool.

Objectives were achieved, allowing the tool to benefit from the advantages of repeatability, reliability, and precision provided by modern-day robotics [11]. A conceptual design can be seen in Fig. 4. The final designed tool is composed by two complementary systems: a material application system composed by mechanical subsystems and actuators, and a vision and security system composed by camera, laser distance sensor, and security micro-switches.

Out of the set of options described in Section 2.1, the option chosen for superficial preparation was compressed air blowing. Hydro-demolition

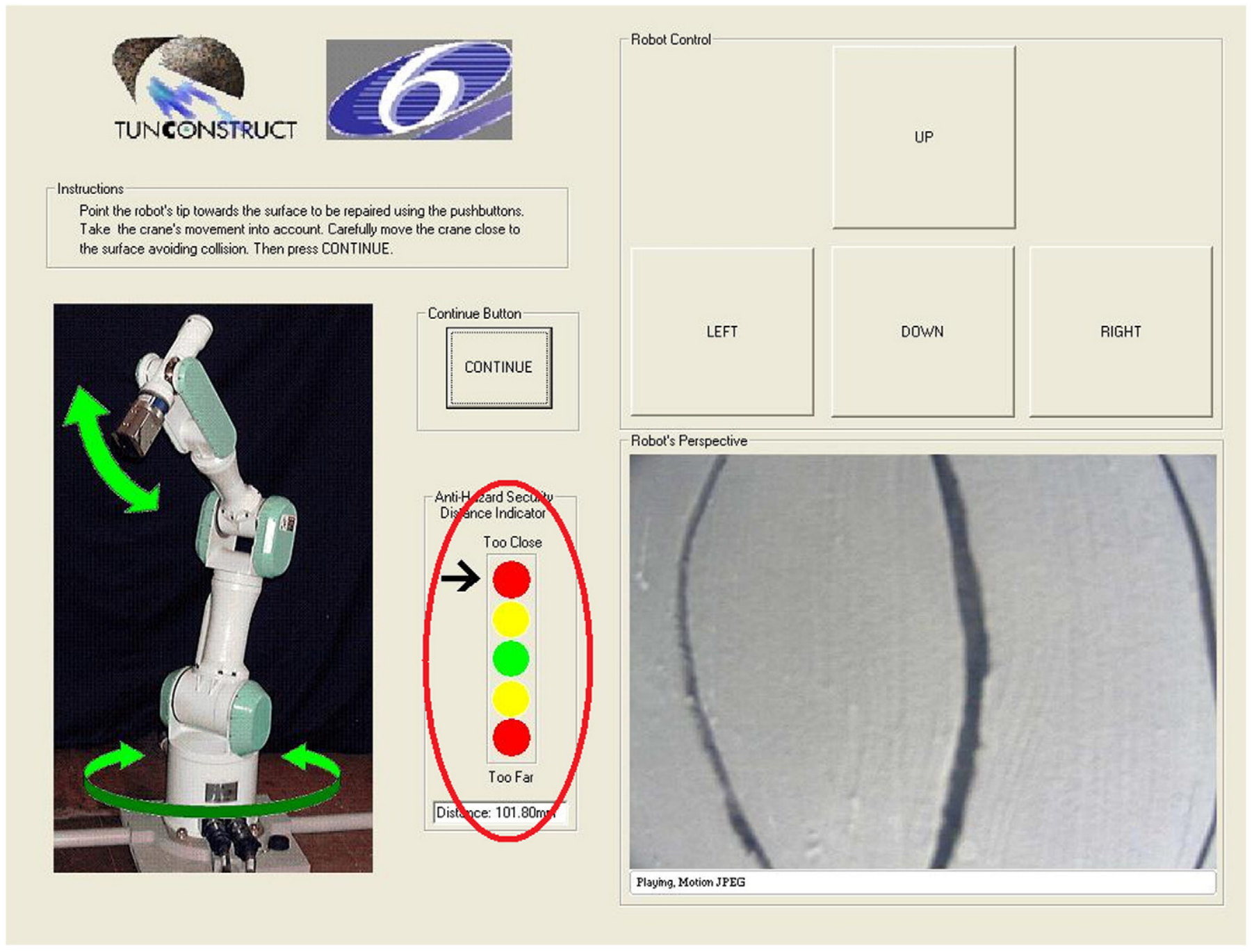

Fig. 9. HMI main screen, w/2 DOF and distance indicator. 
or sand abrasion would have made the availability of water or sand necessary, which would have either incremented the complexity of the system with additional deposits, or created external dependencies. The possibility of sand or water disturbing the flow of traffic would mean that extra protection mechanisms would also have to be devised. As selected and existing composites must be applied on dry and clean surfaces, both hydro-demolition and sand abrasion were discarded to be able to fulfill process time and low-complexity requisites. Compressed air, in any case, would be needed for any of the three cases. It is provided by an on-board compressor mounted on the wheeled vehicle. Most tunnels provide this service, but the compressor is incorporated to increase the flexibility of the system. Air flow is digitally controlled by low power-consumption mini-electrovalves. The nozzle is basically an empty cylinder with one resin input bore $(\phi 5 \mathrm{~mm})$ and many punctures $(\phi 1 \mathrm{~mm})$ for the tunnel side.

The availability of compressed air also led us to one of the system's fundamental breakthroughs: the thrust of the resin towards the tip is triggered by the canalization of compressed air towards the piston of the resin cartridge. This mechanism can be seen in Fig. 5. Therefore, the need of a motor, gear reductions, and additional mechanical components is obviated. This frees the tool from extra weight, size, complexity, and cost.

As mentioned in Section 2.3, commercial FRP is distributed in the form of long rolls; both dry and pre-impregnated rolls are available. The use of pre-impregnated FRP rolls was discarded due to possible undesired FRP-tool interaction caused by the adhesive properties of the composite. Dry FRP must be adhered with epoxy resin at application time. The strips must be pressed against the resin-soaked surface until they are moistened with resin on internal and external sides. This means that the resin must penetrate the FRP and soak the strips internally too. A sophisticated gear-and-roller-set system was designed for the dispensation of dry FRP strips, maintaining the initial single-pass objective. No motor is needed, as motion is achieved through the rotation of a roller by friction with the surface. In the designed system, rollers, gears, and resin dispensation work in conjunction as the tool advances to obtain compact resin-FRP-resin layers mounted on the tunnel's surface. The simultaneity of processes makes the system act as a mini-production line, where the traction roller is always in contact with the dry surface. FRP dispensation and

tool advance velocity radius is adjusted for correctness of functionality. A perspective view of the actual CAD model can be seen in Fig. 6 .

In order to cut the roll of FRP into strips of the desired length, the gear-and-roller system is combined with a guillotine-like cutting mechanism that is activated by relative movements between the tool's internal mobile parts and the tunnel's surface. Linear actuators (pneumatic cylinders) were incorporated to automate the cutting process without having to depend on the robot or manipulator's strength against this surface. This is beneficial for the weaker range of manipulators. Again, the presence of compressed air is used, and the need for motors (cost, weight, and control complexity) is obviated. A linear dispenser, very similar to the one used for air in the superficial preparation process, was designed to spread resin over the tunnel's
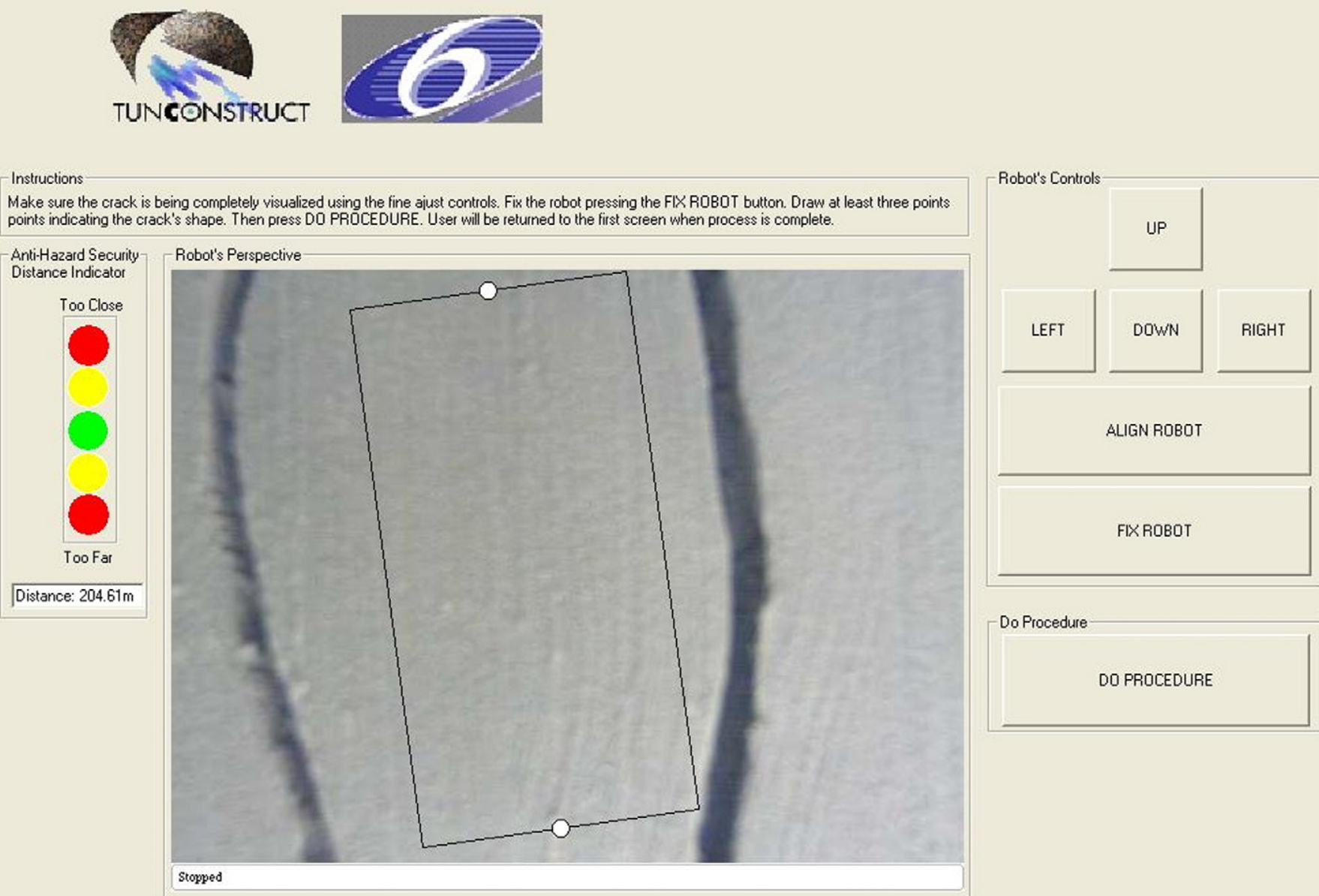

Fig. 10. Intuitive guided HMI screenshots (FRP adhesion). 


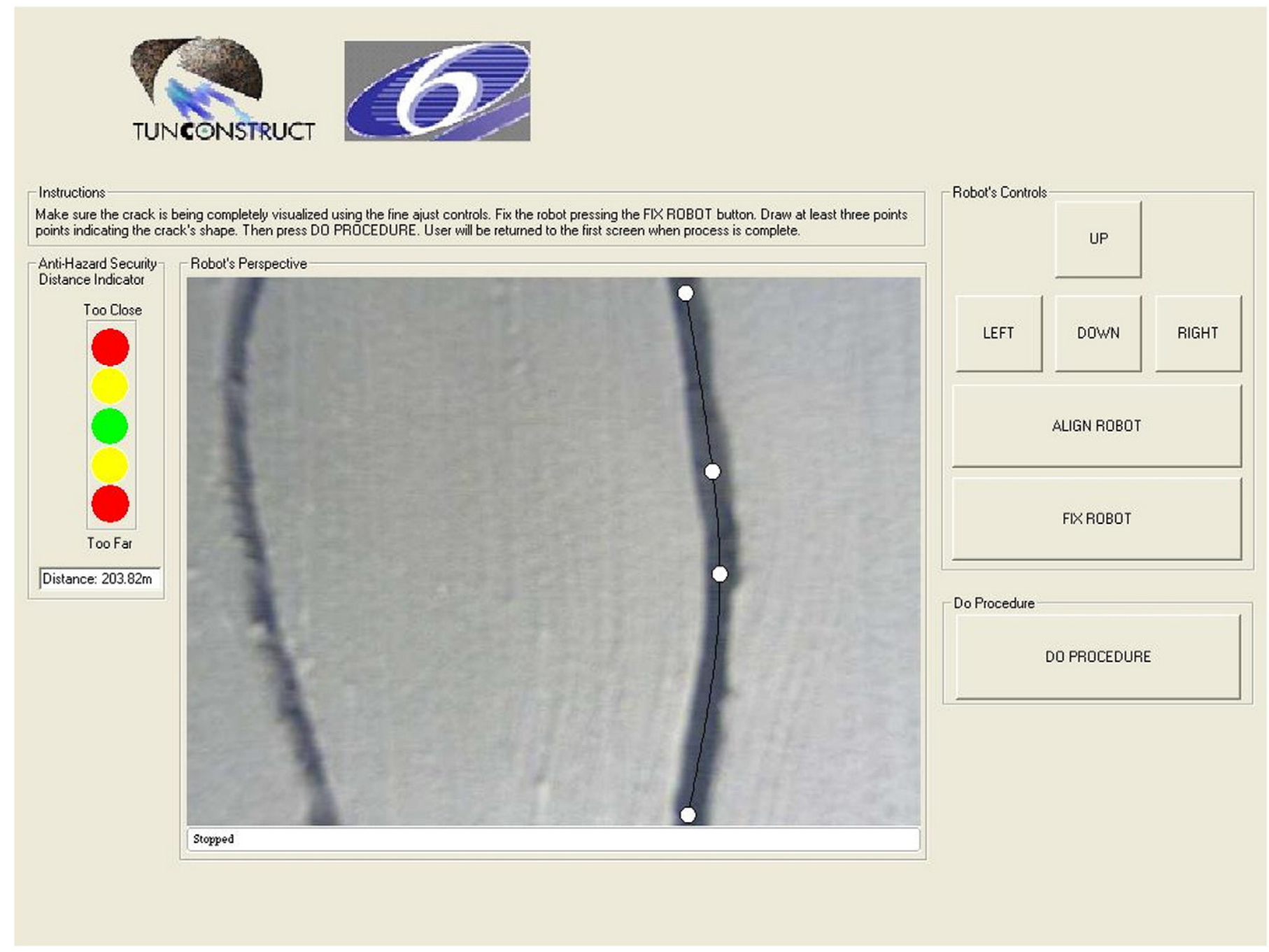

Fig. 11. Intuitive guided HMI screenshots (resin injection).

weakened surface. A transversal section of the final tool design can be seen in Fig. 7, where FRP describes the red trajectory, resin output is set in blue, and additional resin-flattening trowels are circled in orange.

An Axis IP surveillance camera and an ultra-light precision diode laser telemeter sensor are the two main components of the vision system. Sensor ray and the camera's central pixel point are aligned and oriented parallel to the axis of the resin cartridge. Stereoscopic vision was discarded due to its ineffectiveness on homogeneous patterns, such as tunnel concrete surfaces. Homogeneous and uniform patterns create difficulties in the matching process [12]. The IP Surveillance Camera obtains images with a resolution up to $1280 \times 1024$ pixels streaming at 30 frames per second, sufficient for having real-time visual information in the control cabin. Microswitches activated by contact with the tool's mobile parts assure mechanical safety.

\subsection{HMI and control software}

Both HMI and control software were coded entirely in $\mathrm{C}++$, a powerful multi-platform object-oriented programming language. The user-friendly HMI guides the system operator graphically and intuitively through the inspection and identification tasks. The HMI main program flow diagram can be seen in Fig. 8. Following the figure is a more detailed description.
At program initialization subsystems and communications are initialized, and a task selection box is deployed. In the final version of the software, two options are available: superficial preparation and epoxy resin injection, or superficial preparation and FRP adhesion. This value is kept for the rest of the execution of the program, forcing

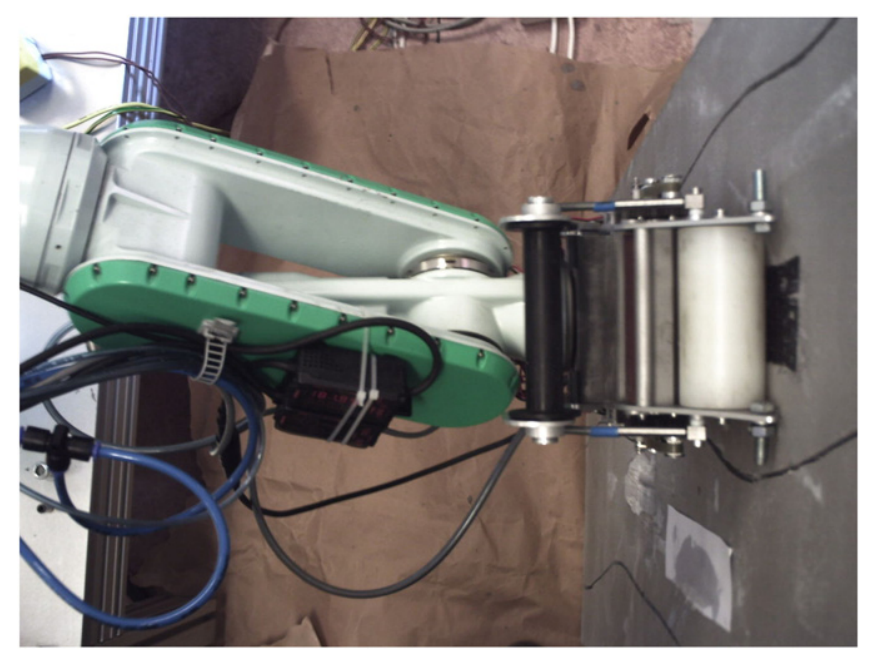

Fig. 12. Laboratory tests at UC3M. 


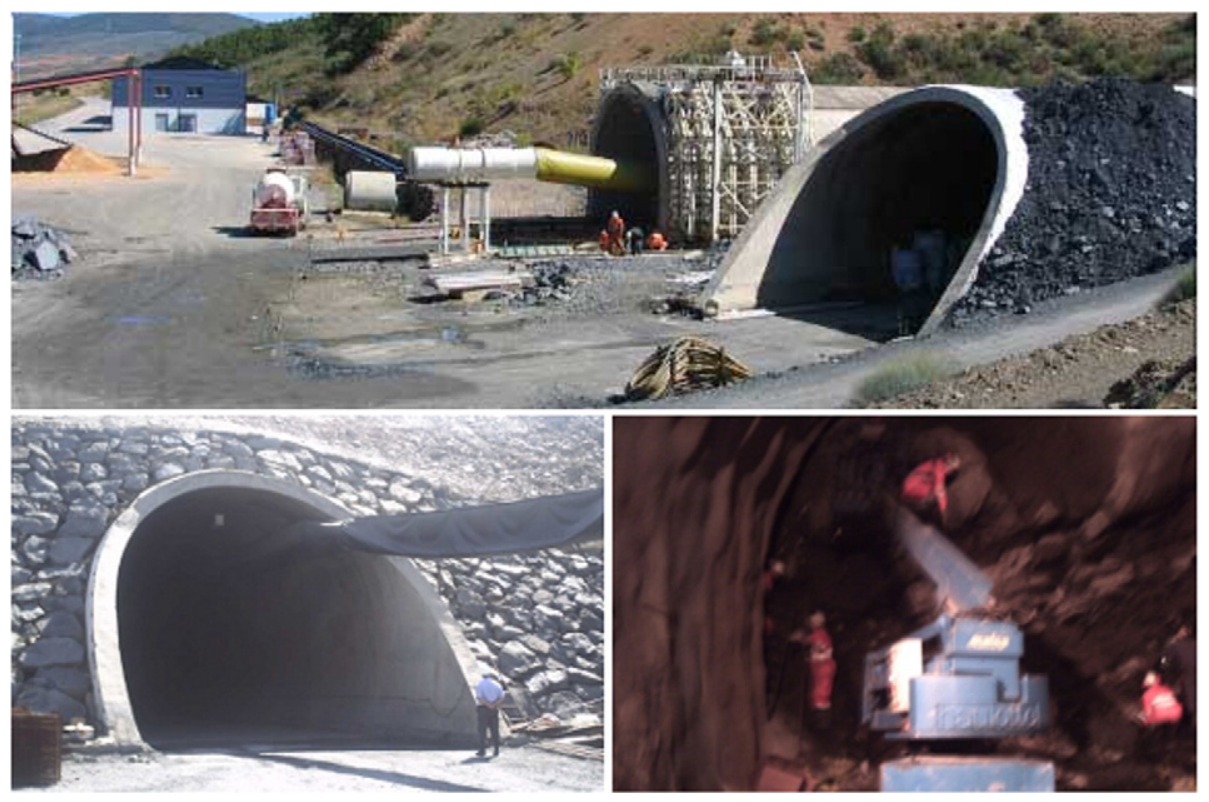

Fig. 13. Tunnels in Fundación Santa Bárbara, at Bembibre (León).

the operator to reboot to confirm having preformed changes on the tool. Once this step is confirmed, the interface passes to the main screen, seen on Fig. 9. Instructions are present at all times throughout the screens. A virtual traffic light (Fig. 9, circled in red) informs the user if the tool tip is too close or too far away from the tunnel surface. The exact measure of the distance from the tool tip to the surface is also presented to the user.

In the first main screen, two degrees of freedom are permitted to the operator for directing the robot: LEFT and RIGHT buttons represent a roll movement of the base joint of the robot, UP and DOWN imply a change of pitch of the end-effector. User is intended to direct the end-effector tool perpendicular to the position of the surface to treat. The operator, when satisfied, presses the CONTINUE button. A zoomed vision of the camera images is displayed, and fine adjustment of the robot tip is performed with the same degrees of freedom. The purpose of this positioning mechanism is to assure the surface is inside of the robot's volume of reach, without putting any part of the system's integrity in risk.

When the operator presses the ALIGN ROBOT button, the robot performs a vertical and a horizontal scan of the surface ( $t$-like shape) and returns to a centered position and orientation. The behavior of the buttons then changes. This change entails that the LEFT and RIGHT buttons move the end-effector sideways (along the length of the tunnel), and the UP and DOWN buttons move the end-effector parallel to the surface (along the spline that was interpolated from the vertical scan of the tunnel). The operator uses these buttons accordingly until he or she is newly satisfied, and then presses FIX ROBOT to finally be allowed to mark the points of interest for trajectory generation. If FRP adhesion was previously selected, the operator marks the limits of the FRP strip to be adhered (Fig. 10). If resin injection was previously selected, a spline-type curve is generated as operator marks points through the interface (Fig. 11). A previsualization of the application can be seen on-screen for either of the two procedures.

The final step for the operator is to press the DO PROCEDURE button. The 2D data introduced by the operator is enough for the system to generate the final 3D robotic trajectories. The system executes these trajectories while coordinating the automatic actuators for task completion in real, non-ideal environments such as tunnels. The operator simply waits for procedures to be performed. On-screen messages inform the operator of the current state of procedure performance. After task accomplishment, the robot safely returns to its initial position, and the interface returns to the main screen.
Internally, software parameters are set in headers, and additional calibration information is calculated at run-time. Reusable spline and geometrical calculation libraries, developed specifically for the robotic application, are integrated into the software. Barrel-type image distortion is corrected using the previously calculated intrinsic parameters of the camera. Communication schemes between HMI and control software are implemented over TCP/IP, the Internet Protocol. This means the operator could, technically, run the HMI software to identify fissures and weakened surfaces from any place in the world. This also means that the use of CPU resources is distributed. One of the PCs is in charge of the control and mathematical transformations for the robot's movements, and the other one manages the user interface. The processor in charge of the graphical interface could also be used for applying machine vision algorithms for the identification of cracks, relieving this task from the user or providing support through augmented reality. Other tasks, such as autonomous control schemes of the crane and wheeled vehicle, could also be controlled from this PC [13].

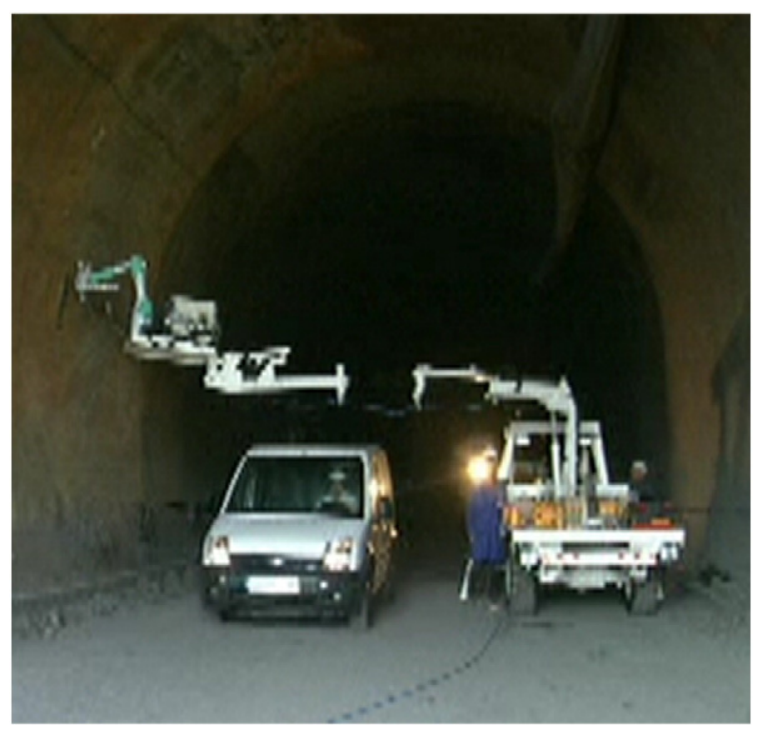

Fig. 14. RATIM system outdoor demonstration. 


\section{Laboratory and field tests}

The RATIM system was first tested indoors, using models manufactured by FRP and epoxy resin providers (Fig. 12). Models were disposed in horizontal, vertical, and intermediate positions to assure material consistency (resin dripping would have been undesirable), robot configuration singularity avoidance [14], as well as correct robot-tool interaction (collision avoidance) $[15,16]$. The robot-tool set was mounted on the wheeled vehicle and tested inside installations of the University.

After having succeeded in proving effectiveness in all of the laboratory tests, the complete integrated system was taken and tested outdoors. Demonstrations were performed in real, non-controlled environments in tunnels in Bembibre, León (Fig. 13). There too, tests provided satisfactory results in terms of human-machine interaction, robot trajectory generation and task execution. A demonstration of how vehicles can pass below the system while process operation is being performed in a functional tunnel can be seen in Fig. 14.

Additionally, the following results from the experiments in the tunnels were obtained.

- A single operator was capable of successfully performing the proposed inspection and maintenance tasks, whereas conventional methods often require from two to five operators.

- The mobile platform could be stabilized at the working position in less than half an hour. A conventional tubular scaffold structure takes several hours to mount at least.

- Performance time of resin application was reduced from a 10 minute average (manual) to less than 7 , mainly due to the high pressure resin output provided by the system. FRP adhesion was reduced from $20 \mathrm{~min}$ to less than 7, in part due to the three-in-one adhesion mechanism also provided by the system.

- Test samples were extracted from the tunnel's surface. Three were results from a manual conventional treatment, and three from the results of the RATIM system (Fig. 15). Analysis indicated no significant difference of the disparity of the stress and strength parameters between the total of six result test samples.

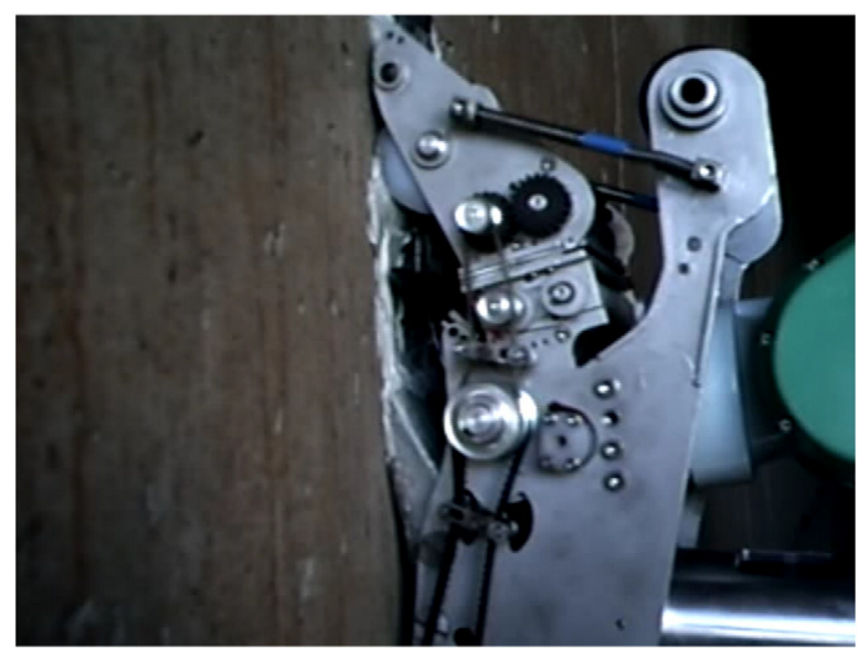

Fig. 15. Close-up of RATIM core tool at demonstration.

\section{Future lines of investigation}

The authors conclude that the synergy of the potential of the RATIM system with other state-of-the-art developments is necessary. Above mentioned and referenced are inspection schemes as those developed for UAVs, and artificial machine vision algorithms for the automatic identification of newly generated cracks on concrete surfaces. Other novel contributions to the system would be automatic tool cleaning systems, perhaps also based on compressed air, and extra stabilizing mechanisms for cranes with booms of lengths similar or longer than those used with the existing system. Dynamic simulations could be performed to quantify the positive impact of the reduction of lane closure on traffic achieved by the system [17]. Furthermore, increase of robustness throughout the design of improved prototypes will always be considered desirable.

\section{Conclusions}

The Robot-Aided Tunnel Inspection and Maintenance System is the first system of its kind. The system has proved its ability to perform operations without need of cutting traffic flow or mounting and dismantling tremendous scaffolds. Developed for inspection, maintenance, and reparation of small fissures and weakened surfaces, it fulfills the philosophy of true preventive maintenance. Reparation of small fissures beforehand will prevent the evolution of these small fissures into great, steep cracks. Reinforcement of weakened structures will prevent solicitations that can provoke the deterioration of the rest of the tunnel's infrastructure. Main characteristics of the RATIM system include reduction of operation times, a quick system and HMI learning curve, and a global increase of commodity and productivity for operator and society. The system has the potential to increase the level of these benefits, as it has proved to reliably automate several steps (but not all) in the performance of the selected tasks.

\section{Acknowledgment}

This work had been funded by the E.U. community under FP6 Project TUNCONSTRUCT. The authors would also like to acknowledge the work of the other partners involved in this project: DRAGADOS, SIKA, and M. Lorenzana.

\section{References}

[1] J.A. Richards, Inspection, maintenance and repair of tunnels: international lessons and practice, Tunnelling and Underground Space Technology 13 (4) (1998) 269-375.

[2] C. Balaguer (2000), Open Issues and Future Possibilities in the EU Construction Automation, IAARC, ISARC '00, Taipei, Taiwan, K21-32.

[3] C. Balaguer, M. Abderrahim, Robotics and Automation in Construction, 2008 ISBN: 978-953-7619-13. IN-TEH.

[4] R. Lackner, H.A. Mang, Cracking in shotcrete tunnel shells, Engineering Fracture Mechanics 70 (2003) 1047-1106.

[5] R. Capozucca, Damage to reinforced concrete due to reinforcement corrosion, Construction and Building Materials 5 (5) (1995) 295-303.

[6] A. Nanni, N.M. Bradford, FRP jacketed concrete under uniaxial compression, Construction and Building Materials 9 (2) (1995) 115-124

[7] S. H. Rizkalla, J. P. Busel, C. E. Bakis, P. N. Balaguru et al. (2005), Guide for the Design and Construction of Externally Bonded FRP Systems for Strengthening Concrete Structures, Reported by ACI Committee 440.

[8] S. Lee, M. Gil, K. Lee, J. Lee, C. Han, Design of a ceiling glass installation robot, IAARC, ISARC '07 Vol. 2 (4) (2007) 247-252.

[9] R. Aracil, C. Balaguer, M. Ferre, M. Buss, C. Melchiorri, Advances in Telerobotics (Editors), Springer Tracts in Advanced Robotics (STAR), vol. 31, 2007, ISBN: 9783-540-71363.

[10] C.W. Kennedy, J.P. Desai, Modeling and control of the Mitsubishi PA-10 robot arm, IEEE/ASME Transactions on Mechatronics Transactions 10 (3) (2003) 263-274.

[11] A. Barrientos, L.F. Peñin, C. Balaguer, R. Aracil, Fundamentos de robótica, McGrawHill84-481-0815-9, 2007.

[12] P. Foggia, J.M. Jolion, A. Limongiello, M. Vento, A new approach for stereo matching in autonomous mobile robot applications, International Joint Conference on Artificial Intelligence (2007) 2103-2108.

[13] C. Balaguer, A. Giménez, J.M. Pastor, V.M. Padrón, M. Abderrahim, Unmanned vehicles for aerial, ground and naval military operations, Elsevier, 2002. pp. 161-166, NATO RTO series, No. 52.

[14] C. Balaguer, Robótica y Automatización, Fundación COTEC, ISBN: 84-95336-61-8, 2006.

[15] L. Moreno, S. Garrido, C. Balaguer, Ingeniería de Control. Modelado y Control de Sistemas Dinámicos, ISBN: 84-344-8055-7, 2003, Ariel.

[16] C. Balaguer, R. Aracil, A. Barrientos, Planning collision-free paths in 3D partially known environment, Advanced Robotics 9 (1) (1995) 15-27.

[17] S. Hwang, Dynamic simulation for assessment of impact of highway lane closure on traffic, IAARC, ISARC '09 (2009) 562-569. 